\title{
SHIPS STEERING AUTOPILOT DESIGN BY NOMOTO MODEL
}

\begin{tabular}{|l|l|l|}
\hline Pradeep Mishra & Dr. S K Panigrahi & Lt Cdr Swarup Das \\
M.Tech student & Professor and HOD & Faculty \& Project Guide \\
Mechanical Engg Dept & Mechanical Engg Dept & MILIT \\
DIAT (DU),Pune & DIAT (DU),Pune & \\
\hline
\end{tabular}

\section{ABSTRACT}

Ships manoeuvring can be automated by using the autopilot system. The marine autopilot system design is based on the mathematical model of steering dynamics. Here in the present paper a study on Nomoto model has been undertaken for its selection for the ships steering dynamics. Choice of selection of the model with respect to fundamental properties of first and second order models has been considered. Effectiveness of the models has been assessed on the basis of main properties of Nomoto model i.e. controllability, observability, identifiability. Further, reasonability of selecting state space model and the transfer function model for the study of different properties has been explained. It is proven that the first order model is controllable and observable whereas the second order model is conditionally controllable. Zero appearing in the transfer function model is found responsible for the overshot behaviour which indicates that the selection of second order model is suitable if the overshoot behaviour has to be studied. First and second order model are identifiable with the ill conditioning problem associated with the latter. hence the first order model is suitable for autopilot applications. Model reductions from fourth order to second and then first order model has been undertaken describing sway-yaw-roll dynamics and bode plots for these models are drawn to show the changes in frequency response due to model simplification.

\section{INTRODUCTON}

This paper is concerned with fundamental properties like controllability, observability, identifiability of Nomoto first and second order model. State space model for Nomoto first and second order model has been derived and solved to find controllability, obeservability because state space model represent non controllable as well as non observable modes along with the observable and controllable modes whereas the transfer function model represents only controllable and observable modes, non controllable and non observable modes are cancelled in transfer function model. Subsequently the system overshoot with respect to Nomoto second order model is explained. Overshoot is caused due to sway coupling effect on yaw rate which is represented by zero and a high frequency pole in Nomoto second order model i.e. $(1+\mathrm{T} 3 \mathrm{~S})$ and $(1+\mathrm{T} 2 \mathrm{~S})$.However the ill conditioning problem due to near cancellation of zero and poles $\mathrm{T} 3 \approx \mathrm{T} 2$ makes second order model less preferable for autopilot design.

In this paper an alternative approach is suggested for adaptive autopilot system which comprises of keeping the zero of second order model which is important for the study of overshoot phenomenon at the same time keeping T3 fixed and varying $\mathrm{K}, \mathrm{T} 1, \mathrm{~T} 2$ to avoid the ill conditioning problem .further with the help of Bode plots for forth ,second, first order model it is proven that why simplification of fourth order model to second order model is not significant because the plots are almost similar except humps in the fourth order 
plot. That means coupling effect of roll mode on yaw motion is negligible but effect of sway couple on yaw motion which is represented by second order bode plot cannot be neglected. Step input response to Nomoto models is also studied.

\section{SHIP STEERING DYNAMICS MODEL SIMPLIFICATION}

Ship response in waves is considered as 6 degree of freedom motion in space. For manoeuvring study 3 dof motion namely surge ,sway, yaw is considered but for heavy vessels effect of roll cannot be neglected hence our study will revolve around 4 dof motion description namely surge, sway, yaw and roll.

Here fourth order transfer function relating yaw rate to rudder angle is derived. Further simplification to second order and first order model is also described

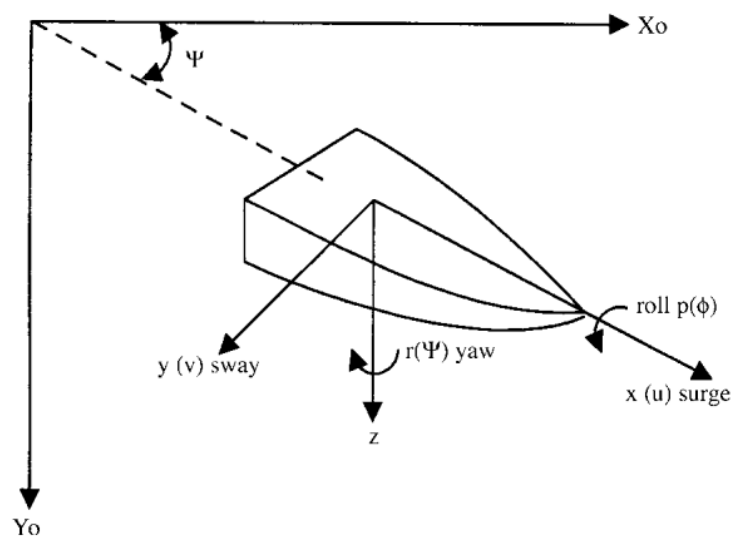

Figure 1 Sway-yaw-roll motion coordinate system.

$m(\dot{v}+u 0 r)=Y v^{\prime}{ }^{\prime}+Y_{V} V+Y \phi \phi+Y \dot{\mathrm{p}} \dot{\mathrm{P}}+Y_{P} P+Y_{r} r+$ $Y_{\dot{r}} \dot{r}+Y_{\delta} \delta$

$I x \dot{\phi}=K P P+K \dot{\mathrm{p}} \dot{\mathrm{P}}-m g \bar{G} \bar{M} \phi+K V V+K$ v́v $+K r r+$ $K \dot{r} \dot{r}+K \delta \delta$
$I z \Psi \cdot=N_{r} r+N_{\dot{r}} \dot{r}+\mathrm{N} \phi \phi+\mathrm{NPP}+N \dot{\mathrm{p}} \dot{\mathrm{P}}+N_{V} V+$ $N v \dot{v}+N_{\delta} \delta$

where $Y V, Y v ́, \ldots$, indicate the hydrodynamic

coefficients; for instance, $Y V$ indicates the derivative of the sway force $Y$ to the sway speed $V$ evaluated at the reference condition; $m$ is the mass of the ship; $I X$ is the moment of inertia about the $\mathrm{X}$-axis; $I Z$ is the moment of inertia about the $z$-axis; $V$ is the sway speed; $u$ is the surge speed; $r$ is the yaw rate; $\Psi$ is the heading angle defined by $\psi=r ; p$ is the roll rate; $\varphi$ is the roll angle defined by $\varphi=p$ and $G M$ is the metacentric height, which indicates the restoring capability of a ship in rolling motion.

Taking the Laplace transform of Eqs. (1)(3) and rearranging, we have

$$
\begin{aligned}
& a_{1} V=a_{2} \phi+a_{3} r+a_{4} \delta \\
& b_{1} \phi=b_{2} V+b_{3} r+b_{4} \delta \\
& c_{1} r=c_{2} V+c_{3} \phi+c_{4} \delta \\
& \text { where } \\
& a_{1}=\left(m-Y_{V}\right) S-Y_{V} \\
& a_{2}=Y \dot{P} S^{2}+Y P S+Y_{\phi} \\
& a_{3}=Y_{\dot{r}} S+Y_{r}+m u_{0} \\
& a_{4}=\mathrm{Y} \delta \\
& b_{1}=(I X-K \dot{P}) S^{2}-K P S+m g \bar{G} \bar{M} \\
& b_{2}=K \dot{V} S+K V \\
& b_{3}=K \dot{r} S+K r
\end{aligned}
$$


$b_{4}=K \delta$

$c 1=\left(I z-N_{r}\right) S-N_{r}$

$c 2=N \dot{V} S+N V$

$c 3=N \dot{P} S^{2}+N P S+N \phi$

$c 4=N \delta$

After eliminating the sway speed $V$ and roll angle $\phi$ from Eqs. (4)-(6), the following transfer function relating the yaw rate $r$ to the rudder angle $\delta$ can be obtained:

$\underline{\mathrm{r}}=\underline{\mathrm{a} 1(\mathrm{~b} 1 \mathrm{C} 4+\mathrm{b} 4 \mathrm{C} 3)+\mathrm{a} 2(\mathrm{~b} 4 \mathrm{C} 2-\mathrm{b} 2 \mathrm{C} 4)+\mathrm{a} 4(\mathrm{~b} 1 \mathrm{C} 2+\mathrm{b} 2 \mathrm{C} 3)}$

$\bar{\delta} \quad \mathrm{a} 1(\mathrm{~b} 1 \mathrm{C} 1-\mathrm{b} 3 \mathrm{C} 3)-\mathrm{a} 2(\mathrm{~b} 2 \mathrm{C} 1+\mathrm{b} 3 \mathrm{C} 2)-\mathrm{a} 3(\mathrm{~b} 1 \mathrm{C} 2+\mathrm{b} 2 \mathrm{C} 3)$

It can be easily verified that the numerator of Eq.19 is third order in S, while the denominator is fourth order in S. Hence, Eq. (19) can be expressed in the following form

$\frac{r}{\delta}=\frac{K\left(1+T_{3} S\right)\left(S^{2}+2 \eta \omega S+\omega^{2}\right)}{\left(1+T_{1} S\right)\left(1+T_{2} S\right)\left(S^{2}+2 \xi \omega_{n} S+\omega^{2}{ }_{n}\right.}$

where the quadratic factors are due to the coupling effect from the roll mode on the yaw rate. The zero $(1+T 3 S)$ and the pole $(1+T 2 S)$ are due to the coupling effect from the sway mode on the yaw dynamics. If the roll mode is neglected, Eq. (20) can be further reduced to the following form

$\frac{r}{\delta}=\frac{K\left(1+T_{3} S\right)}{\left(1+T_{1} S\right)\left(1+T_{2} S\right)}$

Eq. (6) is known as the second order Nomoto model, where $K$ is the static yaw rate gain, and $T 1, T 2$ and $T 3$ are time constants. In practice, because the pole term $(1+T 2 S)$ and the zero term $(1+T 3 S)$ in Eq. (21) nearly cancel each other, a further simplification of Eq. (21) can be done to give the first order Nomoto model

$\frac{r}{\delta}=\frac{K}{(1+T S)}$

Where

$\mathrm{T}=\mathrm{T} 1+\mathrm{T} 2-\mathrm{T} 3$

First order Nomoto model is widely employed in autopilot design and yaw dynamics which is characterised by parameters $\mathrm{K}$ and $\mathrm{T}$ can be determined by manoeuvring tests. Through first order Nomoto model a transfer function relating ships heading $(\Psi)$ to rudder angle $(\delta)$ can be easily calculated by adding $1 / \mathrm{S}$ to transfer function model.

\section{CONTROLLABILITY \& OBSERVABILITY OF NOMOTO MODELS}

Fundamental properties of Nomoto first order model has been assessed here wrt state space model because it represents uncontrollable as well as unobservable modes whereas identifiability property is assessed wrt transfer function model.

Eq (22) can be expressed in time domain as-

$\mathrm{Tr}+\mathrm{r}=\mathrm{K} \delta$

With the notation

$\psi \cdot=r$

$T \Psi+\Psi=K \delta$

Eq 24 and 25 can be arranged in the standard state space form

$x=A x+B u$ 
$y=C x$

where

$x=[\Psi ; r]^{\wedge} T$

$u=\delta$

$y=\Psi$

and

$\mathrm{A}=\left|\begin{array}{cc}0 & 1 \\ 0 & -1 / T\end{array}\right|$

$B=[0 ; K / T]^{\wedge} T$

$C=\left[\begin{array}{ll}1 & 0\end{array}\right]$

According to linear system theory, the system defined by Eqs. (12) is controllable if the following matrix $U$ is of full rank

$U=\left[\begin{array}{ll}B & A B\end{array}\right]$

$=\left|\begin{array}{cc}0 & K / T \\ K / T & -K / T^{2}\end{array}\right|$

and the system is observable if the following matrix $V$ is of full rank

$\mathrm{V}=[\mathrm{C} \mathrm{CA}]^{\wedge} \mathrm{T}$

$=\left[\begin{array}{ll}1 & 0 \\ 0 & 1\end{array}\right]$

Following can be observed from above

1. First order model is controllable and observable. Here controllability means that ships heading and rate of turn can be controlled via application of rudder.

2. Observability indicates that system states ships heading and rate of turn can be obtained by measured data.

3. Identifiability represents that the parameters $\mathrm{K}$ and $\mathrm{T}$ can be determined from i/p (rudder angle) and o/p (yaw rate) which is equivalent to fitting first order model to rudder angle and yaw rate to find $\mathrm{K}$ and $\mathrm{T}$. Hence online estimation of model parameters $\mathrm{K}$ and based on rudder angle and yaw rate is possible and adaptive control strategy can be implemented.

Similar to the discussion about first order model, for the second order model sway to rudder transfer function can be achieved by neglecting the roll mode then subsequently eliminating the yaw rate $r$ from eq (1) and (3)

$\frac{V}{\delta}=\frac{K v\left(1+T_{v} S\right)}{\left(1+T_{1} S\right)\left(1+T_{2} S\right)}$

where $\mathrm{Kv}=$ static sway gain coefficinet $\mathrm{Tv}=$ sway time coefficient

4. If the system is controllable then state variables $\Psi, \mathrm{r}, \mathrm{V}$ should be able to move independently via application of rudder $\delta$.It is thus inferred that for the system to be controllable $\mathrm{Tv} \neq \mathrm{T}$.

5. It can be easily verified that the system is observable that means that all the states $(\Psi, r, v)$ can be reconstructed from the measured heading angle $\Psi$.

6. For identifiability K, T1,T2,T3 should be able to determined via $\delta$ and r.However if $\mathrm{T} 2=\mathrm{T} 3$ zero and pole will cancel each other and ill conditioning problem will occur. Hence for identifiability $\mathrm{T} 2 \neq \mathrm{T} 3$.

\section{BEHAVIOUR OF SYSTEM OVERSHOOT}

Effect of zero term $(1+\mathrm{T} 3 \mathrm{~S})$ on second order Nomoto model by varying values of T3 and keeping T1,T2,K fixed and applying unit step response has been 
studied here. Overshoot is observed when T3 value is higher, for low values of T3 overshoot are not visible. In order to study overshoot behaviour the second order Nomoto model is employed.

In second order model, the overshoot is visible when the zero is on right side of poles and near the imaginary axis. From the equations above and unit steep response curves it is evident that the overshoot is due to sway coupling effect on yaw rate. It can be said that the first order model is relatively simple, doesn't have ill conditioning problem and applied for small rudder angle yaw dynamics, it requires identification of only two parameters hence it is the first choice for

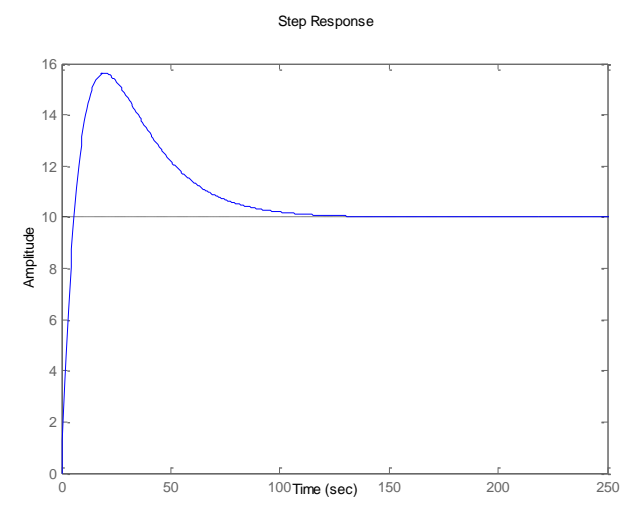

autopilot design.

Figure 2 Unit step response T3=500

Second order Nomoto model is employed where overshoot phenomenon due to large rudder angle turning manoeuvre is to be studied. however it creates an ill conditioning problem due to near cancellation of zero and a high frequency pole.



Figure 3 Unit step response T3=250

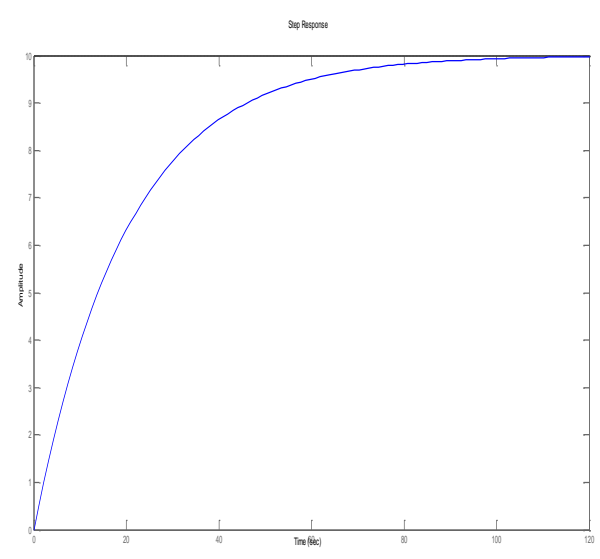

Figure 4 Unit step response T3=100

\section{MODEL REDUCTION AND BODE PLOTS}

Here an alternative approach is suggested for an adaptive autopilot implementation basing upon the second order Nomoto model. Since the zero of the transfer function helps better describing the yaw dynamic overshoot behaviour, its structure is retained and the parameter is fixed at a value determined off-line from inputoutput experiment data. A fourth order linear state space model representing the sway-yaw-roll modes of motion will be used as the nominal model in constructing 


\section{ELK}

Asia Pacific Journals

the corresponding yaw to rudder transfer function. Further simplification to the second order Nomoto model and the first order Nomoto model will also be presented. Using MATLAB, the transfer function of the referred state space model, from $i / p$ rudder to the output yaw rate $r$ is obtained as

$\frac{r}{\delta}=\frac{0.0033 \mathrm{~S}^{3}-0.0004 \mathrm{~S}^{2}-0.0002 \mathrm{~S}-0.000079}{\mathrm{~S}_{4}+0.1913 \mathrm{~S}^{3}+0.0705 \mathrm{~S}^{2}+0.0069 \mathrm{~S}+0.0001}$

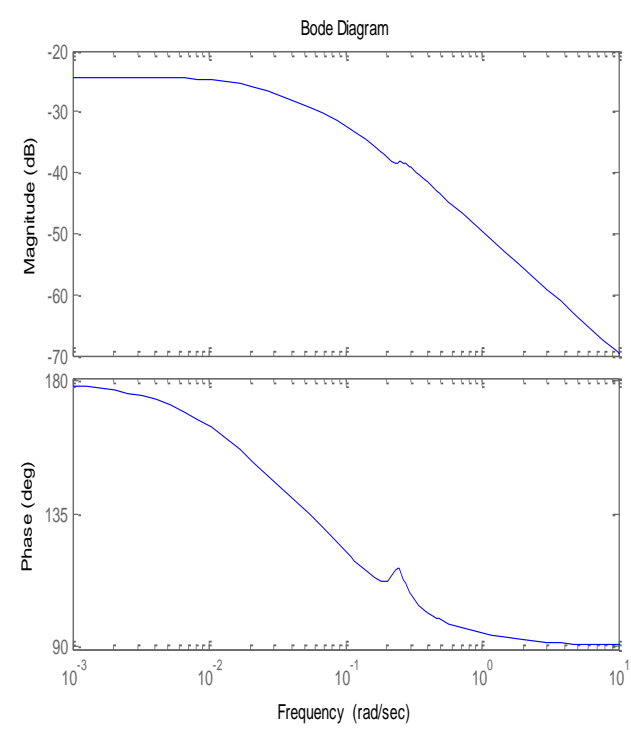

Figure 5- Fourth order model

By neglecting the roll mode the following transfer function can be obtained and fourth order model is reduced to second order model.

$\frac{r}{\delta}=\frac{0.0033 \mathrm{~S}-0.00015}{\mathrm{~S}^{2}+0.1213 \mathrm{~S}+0.00304}$
ELK Asia Pacific Journals - Special Issue ISBN: 978-81-930411-4-7

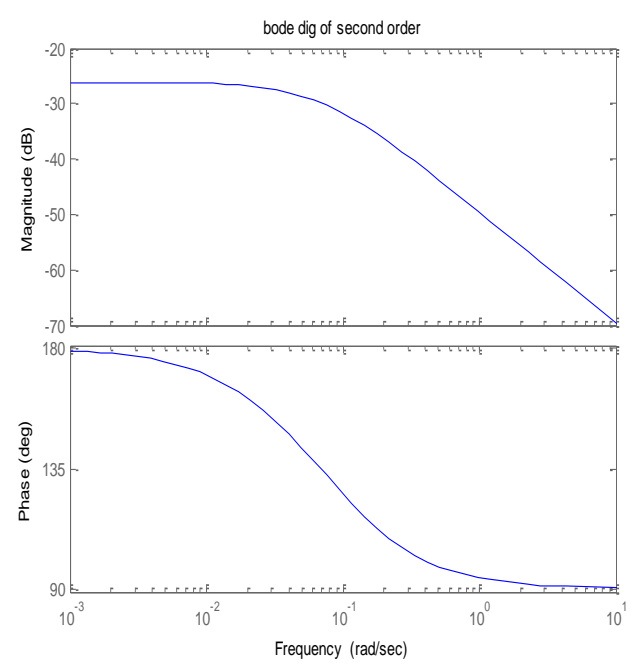

Figure 6 Second order model

further by neglecting the sway coupling effect on yaw rate ,resulting transfer function is reduced to first order model

$\frac{r}{\delta}=\frac{0.049}{1+17.78 \mathrm{~S}}$

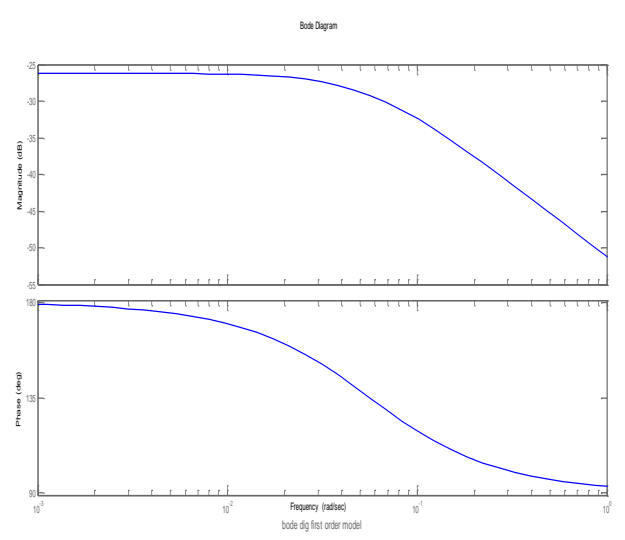

Figure 7 First order model

Bode plots representing frequency domain yaw response and comprising magnitude and phase plots for above mentioned transfer functions are drawn wrt fourth, second, first order model. 
Plots for fourth and second order models are almost similar except the presence of humps in the fourth order model plot. However the first order model plot is significantly different than the first order plot in the magnitude and phase. Based on the observation of above figures, it can be concluded that the effects of model reduction from fourth order to second order is not significant ;namely the coupling effects of roll mode on yaw motion is negligible. However simplification of second order model to first order model poses serious challenges and throws implication of neglecting effect of sway couple on yaw rate which is a very significant aspect and hence cannot be undermined.

Hence it is justified to use second order Nomoto model to represent the behaviour of fourth order model.

\section{CONCLUSION}

The first order model is relatively simple, doesn't have ill conditioning problem and applied for small rudder angle yaw dynamics, it requires identification of only two parameters hence it is the first choice for autopilot design, Second order Nomoto model is employed where overshoot phenomenon due to large rudder angle turning manoeuvre is to be studied. Since the second order Nomoto model includes the coupling effect from sway to yaw mode, it introduces a zero and high frequency pole into the transfer function which contribute in the overshoot tendency. However the ill conditioning problem with the second order model due to near cancellation of zero and pole prevail over the improvements gained in the modelling potential. An approach that retains the zero and at the same time avoids the ill conditioning problem has been proposed.
The state space counterparts of the first order model is found to be controllable and observable, hence the state feedback and output feedback controllers can by successfully implemented. The state space counterpart of second order model is observable but conditionally stable (when $\mathrm{TV}=\mathrm{T}$ ). Bode plots with respect to model simplification from fourth order to second and first order are depicted to show the errors and differences due to model simplification.

\section{REFERENCES}

1. Nomoto, K., Taguchi, K., Honda, K. and Hirano, S., "On the Steering Quality of Ships," International Shipbuilding Progress, Vol. 4, pp. 354370 (1957).

2. Norrbin, N.H., "On the Design and Analysis of the Zig-Zag Test on Base of Quasilinear FrequencyResponse," Technical Report No. B140-3, The Sweden State Shipbuilding Experimental Tank (SSPA), Gothenburg, Sweden (1963).

3. Astrom, K.J. and Kallstrom, C.G., "Identification of Ship Steering Dynamics," Automatica, Vol. 12, pp. 922 (1976).

4. Hwang, W.Y., "Cancellation Effect and Parameter Identifiability of Ship Steering Dynamics," International Shipbuilding Progress, Vol. 26, No. 332, pp. 90-120 (1982).

5. Zhou, W.W., Cherchas, D.B. and Calisal, S., "Identificationof RudderYaw and Rudder-Roll Steering Modelsby Using Recursive Prediction Error Techniques", Optimal Control 
Application and Methods, Vol. 15, pp.101-114 (1994).

6. Fossen, T.I., "Guidance and Control of Ocean Vehicles," John Wiley and Sons, NY (1994).

7. Tzeng and Lin "Adaptive ship steering autopilot design with saturating and slew rate limiting actuator" International Journal of Adaptive Control And Signal Processing (2000)

8. Viorel Nicolau "The Influence Of The Ship's Steering Machine Over Yaw And Roll Motions" The Annals Of "Dunarea De Jos" University Of Galati (2003)

9. L. Morawski, J. Pomirski and A. Rak "Design Of The Ship Course Control System" International Design Conference - (2006)

10. Tristan Perez "Ship Motion Control" Springer-Verlag London Limited (2005)

11. S.Hammoud "Ship motion control using multi controller structure" Journal of Maritime Research (2011) 